\title{
Perspectives of Using Spin Waves for Computing and Signal Processing
}

\author{
György Csaba, Ádám Papp \\ Center for Nano Science and Technology, University of Notre Dame and Faculty for Information Technology and Bionics Pázmány Péter \\ Catholic University \\ Wolfgang Porod \\ Center for Nano Science and Technology, University of Notre Dame
}

\begin{abstract}
Almost all the world's information is processed and transmitted by either electric currents or photons. Now they may get a serious contender: spin-wave based devices may just perform some information processing tasks in a lot more efficient and practical way. In this article, we give an engineering perspective of the potential of spin-wave-based devices. After reviewing various flavors for spin-wave-based processing devices, we argue that the niche for spin-wave based devices is low-power, compact, high-speed signal processing devices, where most traditional electronics shows poor performance.
\end{abstract}

Keywords: Magnonics, spin waves, logic devices, microwave processing

\section{Introduction: flavors of spin-wave based devices}

It is widely acknowledged that Moore's law - the exponential scaling of semiconductor performance in the past ${ }^{35}$ few decades - is about to come to an end [1]. The research 5 field for new computing devices is wide open for disruptive ideas. This presents a unique opportunity for studying fundamentally new device concepts - among them devices that use the spin degree of freedom for representing and transmitting information [2]. There are many pos10 sible approaches for spin-based processing: one may use single-domain magnetic states and their interactions [3] or electrically-interconnected devices with magnetic layers lying at the heart of their operation. A less straightforward way is to think about a dynamic computing device, one 15 that uses collective excitations of a spin-lattice for information processing. Such devices are the subject of this article.

Spin waves (magnons) are rather different from electromagnetic waves, but small-amplitude spin waves dis-

20 play interference phenomena very similar to photonic interference. It is instructive to draw an analogy between electromagnetic waves and spin waves, as most spin-wavebased information processing devices (magnonic devices) can find their origin in a photonic structure - and proposed 25 magnonic devices face challenges similar to their photonic counterparts [4].

Just as in the case of photonic devices, one may follow two distinct routes toward information processing [5]. One route aims to replicate the functionality of electronic tran-

30 sistors and logic gates and follow the footsteps of highly successful digital electronic circuitry. The advantage of this approach is that there is a clear path toward large and complex computing systems. Boolean circuitry, however, may not be a very good fit to spin-wave based devices and it is not at all clear whether spin-wave based Boolean networks will be competitive with highly optimized, CMOSbased computing devices. Photonic logic devices may give a lesson here: despite numerous benefits, the input / output overhead and their relatively large (wavelength-scale) size makes them impractical as a replacement for CMOSbased circuitry.

Non-Boolean device constructs may allow one to exploit the unique benefits of spin waves, i.e. the computational power of interference pattern formation. This is often referred as 'wave-based computing' or 'holographic computing' and in optics, such computational primitives were widely known under the titles 'Fourier optics' or 'Information optics' [6]. Wave computing does not lend itself easily to general-purpose computing tasks, but provides relatively simple and elegant ways for e.g. doing linear filtering, or calculating Fourier transforms [7]. These are important computing primitives and, for example, in image processing algorithms and in neural networks such simple and repetitive transforms may be responsible for the bulk of the power consumption [8].

An additional application of non-Boolean processing (linear transforms) is that these algorithms are key components in microwave processing algorithms (i.e. in the frontend of radio-frequency and telecommunication devices). In such applications, real-time processing is required in the several ten gigahertz frequency range. There are no lowenergy CMOS solutions for these tasks - transistor-based, active circuits consume significant power in order to overcome noise and parasitic effects. We will argue that spin- 
65 wave-based devices make intrinsically good high-frequency signal processors. The use of magnetic materials for this 120 purpose is not at all new [9] [10], but adding spin-wave interference to the toolbox may open new horizons.

There are a number of excellent review articles focusing 70 on spin-wave logic devices or magnonic crystals - the latter being perhaps the most established area of spin-wave based devices. The readers are referred to [11] [12] [13] [14]. The paper of Chumak et. al. [5] is a comprehensive review of spin-wave-based, beyond-Moore devices. In this article, we

75 focus on the less-travelled, but, in our opinion, promising route of non-conventional, non-Boolean device structures.

\section{Crash course on spin waves}

Spin waves (SWs) are wave-like excitations in magnetic materials - the waves propagate by either exchange 80 or dipole interactions between precessing spins. SWs can be understood as particular, wave-type solutions of the time-dependent Landau-Lifshitz-Gilbert equation (LLG) ${ }^{125}$ [12]. The LLG equation describes the dynamics of the $\mathbf{M}(\mathbf{r}, t)$ magnetization distribution, and, under some circumstances, the solution can be approximated as $M_{a} \propto$ $\sin (2 \pi f \mathbf{k} t), M_{b} \propto \cos (2 \pi f \mathbf{k} t), M_{c}=$ const., where $M_{a}$, $M_{b}, M_{c}$ are the $x, y, z$ components of the $\mathbf{M}(\mathbf{r}, t)$ mag- $^{130}$ netization vector, $f$ is the spin-wave frequency and $\mathbf{k}$ is the wave vector. Plane-waves are small perturbations of a uniformly magnetized spin-ensemble. For most practical purposes we are interested in magnetic thin-films.

It is easy to see that depending on the relative orienta- ${ }^{135}$ tion of $\mathbf{M}$ and $\mathbf{k}$, there are different possible propagation modes. From the theory point of view, the most straightforward case is $\mathbf{M} \perp \mathbf{k}$, such as in the case of an out-of plane magnetized thin-film - a particular dispersion relation for this case is shown in Fig. 1a. Spin-wave propaga- ${ }^{140}$ tion is isotropic in this case. An example of an anisotropic in-plane spin wave propagation is given in Fig. 1b. The curves of Fig. 1 are valid only for thin (thinner than a few-ten nanometers) magnetic films, which films are the most relevant for the device applications discussed here ${ }^{-145}$ for thicker films, additional modes may appear.

The dispersion relations show that typical frequencies in the $5-100 \mathrm{GHz}$ range correspond to wavelengths in the ten nanometers - few micrometers range. This perfectly matches the frequency range and size scale where mod-150 ern electronic circuits operate. Spin-wave wavelengths go all the way down to the nanometer range, so spin-wavebased devices (unlike photonic structures) can be scaled all the way down to the sizes of end-of-the-roadmap semiconductor devices. Also, spin-wave wavelengths at microwave frequencies are about six orders of magnitude shorter then ${ }^{155}$ electromagnetic waves - this hints at the possibility of using spin waves for replacing bulky (centimeter-scale) microwave structures.

The high-frequency tail of the dispersion relation in yttrium iron garnet (YIG) reaches the low $\mathrm{THz}$ regime, ${ }^{160}$ where electronic circuitry is more challenging or impossible to design.
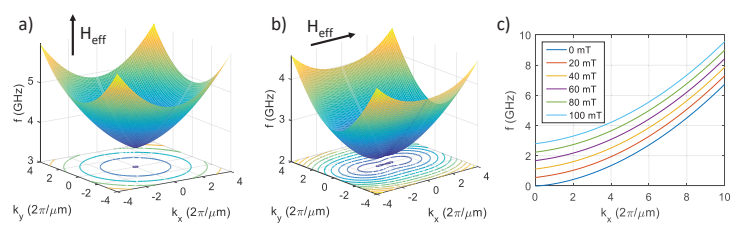

Figure 1: Dispersion relations for spin-wave propagation in YIG. $a$ ) isotropic spin-wave propagation in a perpendicularly magnetized film (b) anisotropic propagation for in-plane SWs $c$ ) magnetic field dependence of the dispersion relation.

Spin waves propagate by two distinct mechanisms: shortwavelength (typically $\lambda<100 \mathrm{~nm}$ ) SWs by the locally strong exchange interactions and long-wavelength SW by dipolar interactions. Fortunately, the ways of creating and manipulating these two SW regions can be done by similar methods, but for most practical purposes one tries to operate in the exchange-dominated, short-wavelength regime.

Another benefit of spin waves is illustrated in Fig. 1c, which shows how the dispersion relation can be altered by the application of a magnetic field. The frequency and wavelength can be tuned to the possible application. Also, one may tune $\frac{d \lambda}{d f}$ i.e. the frequency sensitivity of the wavelength.

Engineering SW dispersion is extensively researched in the framework of magnonic crystals [12] [13].

The propagation length of spin waves for long was considered a problem: due to relatively strong damping and fast decay of the SW amplitude, complex interference patterns were hard to generate in thin-films. Recently, it became possible to reach SW propagation for at least few-hundred wavelength distances [15], which is still short compared to optical waves, but sufficient for many device applications.

Historically, the potential applications of SWs for microwave processing were recognized long ago, and low-loss magnetic materials with engineered spin wave spectrum were used in microwave passive filters, signal-to-noise enhancers and as tunable inductors [10]. In these applications, the magnetic materials were used as a tunable inductive load, but the spatial complexity of SW interference patterns was not exploited. Modern nanotechnologies allow direct access to SW interference patterns, opening up opportunities for new devices.

\subsection{Manipulating spin waves}

Figure 1c shows the sensitivity of the dispersion relation to magnetic fields. Changes in the $\mathbf{H}_{\text {eff }}$ effective magnetic field change the dispersion relation, changing the effective index of refraction for the magnetic thin film (and shifting spin-wave phase).

There are a number of possibilities for engineering the magnetic field landscape on the submicrometer size scale. 
Changing the thickness of the magnetic film changes the effective field and can realize waveguides for spin waves 215 [16]. Another approach relies on breaking the periodicity of a magnonic crystal array [17].

An especially promising idea is to create localized magnetic fields by additional layers of magnetic materials [18][19]. Using Yttrium Iron Garnet (YIG) enables low-loss spin-220 wave propagation, adding metallic nanomagnets on top changes the magnetic field landscape seen by the SWs.

There are more unusual and potentially flexible ways to manipulate spin waves, such as optically [20], or by heat-assisted nanopatterning [21].

Perhaps the most straightforward way to guide SW propagation is patterning the magnetic film. This is straightforward for the case of metallic ferromagnets and it has recently become possible for less fabrication-friendly materials, such as YIG [22].

It is extremely useful if the potential landscape of the magnetic materials can be manipulated in a dynamic, reconfigurable way [23]. Strain control of spin waves [24] could potentially enable reconfigurable steering of spin waves with very low energy.

\subsection{Energetics of spin waves}

A major motivation for spin-based electronics is addressing the power bottleneck of today's microelectronic devices. For static, spin-based logic devices there are strong reasons to believe that they can dissipate very low power during operation [25]. For SW-based devices, much less esearch was done. A simple calculation [26] suggests that ${ }_{225}$ a relatively complex calculation (a 64-point Fourier transform) can be done with a spin-wave wavefront carrying a mere few-ten electronvolts of energy. As the formation of the interference pattern takes place on the nanosecond time scale, the power efficiency of computing based $_{230}$ on spin-wave interference pattern is orders of magnitude higher than that of a Boolean network. Most likely, power consumption of any spin-wave based device is dominated by the input / output apparatus [27]. New physical effects (such as non-local spin injection) could boost high power ${ }_{235}$ efficiencies, at least at the input side [28].

\subsection{Spin waves for transmitting information}

One of the earliest concepts for application of spin waves in microelectronics was based on the recognition that they may provide superior, wideband, short-range interconnections [29] akin to an on-chip photonic structure. This argument was later debated for metallic spin-wave conductors [30] - but it is possible that spin-waveguides made from low-damping magnetic materials significantly

\section{Boolean computing protocols based on spin waves}

Just as in the case of photonic devices, one route to the design of wave-based logic gates is a Mach-Zender

interferometer-like structure. A recent implementation is illustrated in Fig. 2. Spin waves may interfere constructively or destructively, depending on the phase relation of the inputs [32], or possibly due to external, currentinduced fields [33]. The relation between the input phases and the output amplitude (or phase) maps to a majority gate, which is a universal building block for logic devices.

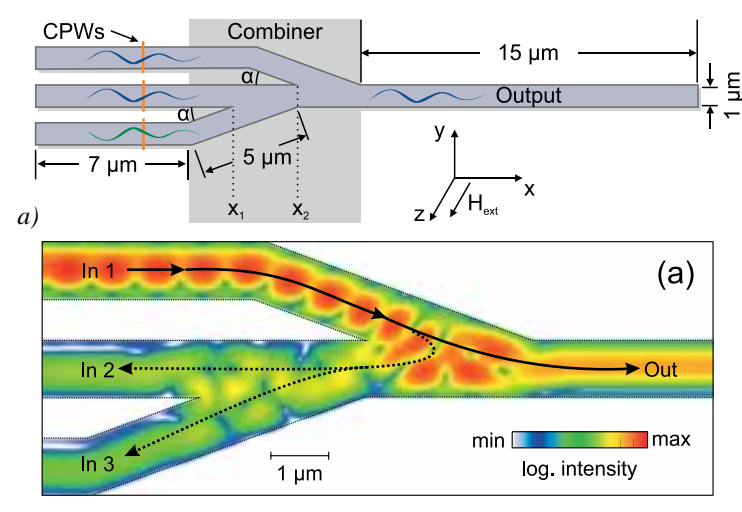

b)

Figure 2: Example of Boolean spin-wave based processing, taken from [32].

A different approach is to realize the magnonic equivalent of a transistor [34], which can be done exploiting the nonlinearity of magnon-magnon scattering.

It is important to keep in mind that the realization of a Boolean computing system requires a lot more than stand-alone logic gates. Some system components (such a beam splitter [35]) were demonstrated, others were not. A useful guide here is the 'five tenets' of computing (see e.g. [36]), i.e. (1) nonlinear device characteristics, (2) complete set of Boolean operators, (3) output of one device can drive the next one, (4) input / output isolation and (5) power gain. The Boolean logic gates demonstrated so far certainly satisfy (1) and (2), but practical implementations to (3), (4) and (5) remain a challenge. It is straightforward to realize gain and isolation using electrical circuitry, but it is likely that a practical computing system should try to minimize the number of magneto-electric conversions and the five tenets should be fulfilled by spin-wave based constructions.

\section{Spin-wave based, non-Boolean devices}

Special-purpose, non-Boolean computing units may avoid the above-mentioned challenges associated with the interconnections of logic blocks. If a non-Boolean computing unit performs a high computing function (i.e. something that is way beyond the function of a simple logic gate) and this requires only relatively few inputs and outputs, then the input / output bottleneck may be less severe. We exemplify the construction of non-Boolean computing blocks 
below. These devices are based on optical computing primcertain optical processing tasks.

Figure $3 \mathrm{a}$ shows a high-level schematics of the spinwave based processor. The device realizes a vector-vector ${ }_{295}$ mapping: electrical inputs generate a spin-wavefront with -defined amplitude and phase distribution and under the influence of local effective field distribution the result of the mapping (computation) is a SW intensity distribution, which is converted to an electrical signal for further 300 processing.

A possible, immensely useful non-Boolean operation is analog Fourier transform. It is well known from optics [6] that a convex lens can generate the Fourier transform of the coherent light distribution. In SW-optics, one can ${ }_{305}$ use a concave (effective) field distribution to generate the same diffraction pattern [37]. Figure 3b) shows the magnetization distribution on the computing layer, as calculated from micromagnetic simulations. The SW lens is a planar structure, i.e. SW propagation in $2 \mathrm{D}$ is used to transform a one-dimensional vector. This particular SW structure

270 was proposed in [37], and a very similar structure was experimentally realized in [38].

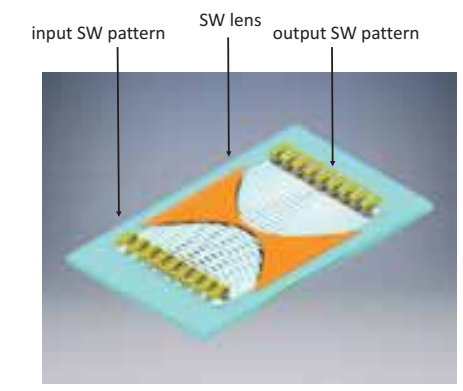

a)

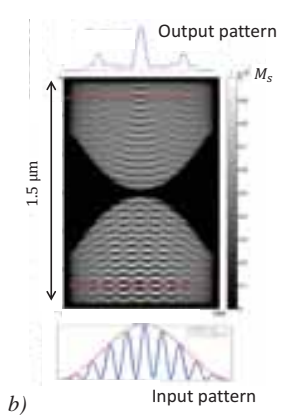

duced and their structure simplified if the Fourier transform of a time-domain signal is of interest [27][43]. This can be done by a spin-wave-based implementation of the Rowland circle spectrograph is shown in Fig. 4. A single microwave stripline (antenna) is run along the serrated edge of a magnetic film. The cogged film edge simultaneously acts as a source of spin waves and as a diffraction grating. Since the diffraction grating is curved, spin waves with different wavelengths are eventually focused into different points along the so-called Rowland circle. If the current of the microwave transmission line is composed of multiple frequency components, those frequency components will generate spin waves with different wavelengths, and the multitude of frequency components will eventually be focused at different points along the output (the so-called Rowland circle). The device acts as a spectrum analyzer - a critically important and ubiquitously used component in signal processing and RF front-ends.

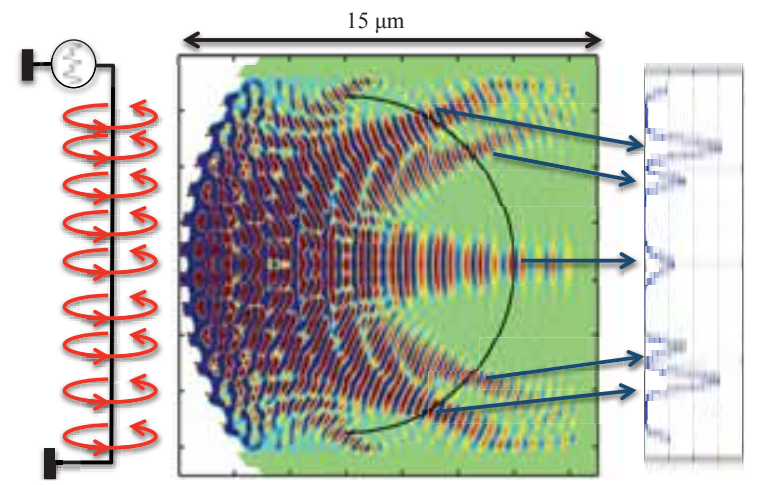

Figure 4: Schematics of a spin-wave based spectrum analyzer. The main component of the device is a curved diffraction grating, shown at the left of the image. Schematics of the I/O structures are overlaid on a micromagnetic simulation of the device. Taken from [27].

These above described 'quasi-optical' devices rely on

Figure 3: a) Spin-wave lens schematics b) Micromagnetic simulation of spin-wave distribution, as shown in [37].

The structure of Fig. 3 is still somewhat heavy on inputs and outputs: a say, 64-point Fourier transform requires 64 analog input points, where spin-wave intensity can be individually controlled and the same number of output, where the SW intensity can be measured and con- ${ }^{315}$ verted to electrical signals. Still, such a device can perform Fourier transforms in a single step, with several gigasamples per second speed and potentially replacing several thousands of individual logic gates [39].

Another approach to non-Boolean vector-vector map- ${ }^{320}$ ping relies on crossbar-like structures of magnetic waveguides [40]. Depending on how the SW phase is altered at the crossings, these devices can also make complex linear transforms between their inputs and outputs in a single step. Elementary steps of holographic pattern matching and prime factorization [41] have been experimentally demonstrated in this scheme. One may construct a variety 325 of interconnection / processing schemes in this spirit [42]. spin-wave interference pattern formation. It is also possible to use spin waves solely as an interconnection between spin-based electronic devices and implement non-Boolean functions this way. For example, spin-wave based synchronization of spin-torque oscillators (STOs) have been proposed for vector-vector distance calculation [44] [45] [46][47], which is another ubiquitously used computing primitive in image processing. There is a large number of ferromagnets SWs could substitute short-range electrical interconnections

\section{Enabling technologies spin-wave devices}

The last few years have seen a tremendous progress in the technologies leading towards spin-wave devices. Perhaps one of the most important of those is the availability of high-quality (low damping) sputtered YIG films [49], [50]. The quality of sputtered YIG films now rivals that of epitaxially-grown YIG, and chances are that such that promising spin-torque building blocks [48], and in metallic 
YIG (a brittle, hard to work with material) can be one day compatible with well-established microelectronic technologies. A major step in this direction is the recent result to385 grow YIG films on silicon substrate [51].

YIG is not the only material that may serve as spinwave channel in SW devices. Cobalt-Iron compounds exhibit very low damping at specific composition [52] - and CoFe would be readily integrable with microelectronic de- -390 vices. The high group velocity of spin waves in CoFe thin films [53] is beneficial for the envisioned high-frequency applications. Barium hexagonal ferrite (BaM) combines low damping and strong perpendicular magnetic anisotropy [54]. Almost all SW-based devices require an external ${ }_{395}$ magnetic field for their operation, but sufficiently strong anisotropy, which is seen in BaM can substitute cumbersome permanent magnets.

\section{The main challenge: magneto-electric interfaces}

As it was argued above, the Achilles's heel of SW-based devices (and, for that matter, most magnetoelectronic devices) is the input / output: the eventual practicality of these devices hinges on whether one can find energyefficient, compact structures to convert electrical signals into spin-wave signals and vice versa.

Spin-wave excitations can be straightforwardly created by the Oersted field of waveguides. This is not a particularly power efficient method - the vast majority of the power pumped in the microwave transmission line is lost on the terminating resistance, and only a few percent of the energy is taken away by the spin waves [27]. Another challenge is the generation of short-wavelength spin waves, which is important in microelectronic applications - the non-localized field distribution of a typical micron-width mission line couples poorly to submicron-width SWs. Alternatively, spin-torque oscillators are excellent sources of short-wavelength SWs in metallic thin-films [55] [56] [57], and they may be used on magnetic insulators as well ${ }^{415}$ [19] [58]. A third possibility is to use magnetostriction only electric field (and no DC current flow) is used for inducing spin-waves. One may use wavelength conversion to squeeze SW wavelength [60] or patterned multilayer struc- ${ }^{420}$ tures [31] or nanoscale domain structures [61] to generate sources.

Spin-hall effect (SHE) -based manipulation of magnetization [62] and spin-pumping [63] are perhaps the most $_{425}$ application-friendly way to generate SWs, especially in magnetic insulators. Spin-pumping materials (such as $\mathrm{Pt}$ ) low-power applications.

Waveguide, STO or SHE based SW generation all typically require currents on the order of milliamperes, and consequently, spin-wavefronts or spin-wave point sources (in case of an STO) can be created by power on the order ${ }_{435}$ of milliwatts. Recent experimental results point toward possibly much more power-efficient ways [28].
SW can be converted to electrical signals by magnetoresistive structures or (in case of magnetic insulators) via antennas or inverse SHE [64]. In all cases one typically expects signals in the few microvolt - few ten microvolts range [27]. DC readout of such signals is straightforward, but requires significant amplification before signals can be sent for further processing. In a practical scenario, however, one should pickup these signals with a significant (few ten megahertz to gigahertz) bandwidth - this requires carefully matched, low-impedance antennas, amplifiers and appropriate mixers [27]. It is expected that the SW readout will dominate the complexity and power consumption of SW-based processing systems.

\section{Outlook}

Spin waves have the potential to make a big hit in future data processing - they can combine the best of electronics and photonics and do it in an on-chip and integrable way. However, copying successful approaches from microelectronics may not be the best way toward spinwave based computing, as the resulting circuits tend to require too many conversions between the electric and magnetic domains, introducing serious complexity and power bottlenecks. Spin-wave-based protocols will shine if complex processing tasks can be made without leaving the spin-wave domain, and construction of devices for nonBoolean computing primitives could be a way toward this goal.

There are many intriguing possibilities we did not even mention in this article: such as using nonlinear effects for computing, quantum circuits [65], or the possibility of using spin waves all the way up to the terahertz range [66].

\section{Acknowledgements}

Our work into spin-wave devices was partially supported by the NSF grant Nanoelectronics Beyond 2020 (NEB 2020) and a gift from Intel Corporation. The authors are grateful to Arpad Csurgay (Pazmany University), Jonathan Chisum and Gary Bernstein (University of Notre Dame), George Bourianoff (Intel corp.), Giovanni Carlotti (University of Perugia) and Mathias Benjamin Jungfleisch (Argonne) for stimulating discussions.

[1] see e.g. IEEE Spectrum: Special report: 50 years of Moore?s law, The glorious history and inevitable decline of one of technology's greatest winning streaks http://spectrum.ieee.org/static/specialreport-50-years-of-moores- law

[2] Kerry Bernstein, Ralph K. Cavin III, Wolfgang Porod, Alan Seabaugh, and Jeff Welser, ?Device and Architecture Outlook for Beyond-CMOS Switches,? Proceedings of the IEEE 98 (12), 2169-2184 (2010).

[3] Csaba et. al.: Nanomagnetic logic: from magnetic ordering to magnetic computing in: CMOS and Beyond: Logic Switches for Terascale Integrated Circuits 1st Edition, Cam- bridge University Press, 2015, Eds: Tsu-Jae King Liu, Kelin Kuhn

[4] Saleh, Bahaa EA, Malvin Carl Teich, and Bahaa E. Saleh. Fundamentals of photonics. Vol. 22. New York: Wiley, 1991. 
[5] Chumak, A. V., V. I. Vasyuchka, A. A. Serga, and B. Hillebrands. "Magnon spintronics." Nature Physics 11, no. 6 (2015): 453-461.

[6] D. G. Feitelson: Optical computing : a survey for computer510 scientists, MIT Press, 1988

[7] Ambs, Pierre. "Optical computing: a 60-year adventure." Advances in Optical Technologies 2010 (2010).

[8] Fang Y, Yashin VV, Seel AJ, Jennings B, Barnett R, Chiarulli DM, Levitan SP. Modeling oscillator arrays for video analytic ap-515 plications. InProceedings of the 2014 IEEE/ACM International Conference on Computer-Aided Design 2014 Nov 3 (pp. 86-91). IEEE Press.

[9] Camley, R. E., Z. Celinski, T. Fal, A. V. Glushchenko, A. J. Hutchison, Y. Khivintsev, Bijoy Kuanr, I. R. Harward, V. Veer-520 akumar, and V. V. Zagorodnii. "High-frequency signal processing using magnetic layered structures." Journal of Magnetism and Magnetic Materials 321, no. 14 (2009): 2048-2054.

10] Ishak, Waguih S. "Magnetostatic wave technology: A review." Proceedings of the IEEE 76, no. 2 (1988): 171-187.

11] Lenk, Benjamin, Henning Ulrichs, Fabian Garbs, and Markus Munzenberg. "The building blocks of magnonics." Physics Reports 507, no. 4 (2011): 107-136.

[12] Klos, Jaroslaw W., and Maciej Krawczyk. "Magnonic Crystals: From Simple Models toward Applications." Magnetic Structures530 of 2D and 3D Nanoparticles: Properties and Applications (2016): 283. in : Levy, J.C.S. ed., 2016. Magnetic Structures of 2D and 3D Nanoparticles: Properties and Applications. CRC Press.

[13] Krawczyk, Maciej, and D. Grundler. "Review and prospects of magnonic crystals and devices with reprogrammable band struc-535 ture." Journal of physics: Condensed matter 26, no. 12 (2014) 123202 .

[14] V V Kruglyak, S O Demokritov, D Grundler. Magnonics. Journal of Physics D: Applied Physics, IOP Publishing, 2010, 43 (26), pp.264001.

[15] Yu, Haiming, O. d'Allivy Kelly, V. Cros, R. Bernard, P. Bortolotti, A. Anane, F. Brandl, R. Huber, I. Stasinopoulos, and D. Grundler. "Magnetic thin-film insulator with ultra-low spin wave damping for coherent nanomagnonics." Scientific reports 4 (2014).

16] Demidov, V. E., S. Urazhdin, A. Zholud, A. V. Sadovnikov, and S. O. Demokritov. "Dipolar field-induced spin-wave waveguides for spin-torque magnonics." Applied Physics Letters 106, no. 2 (2015): 022403.

[17] Grundler, Dirk. "Spintronics: Nanomagnonics around the cor-550 ner." Nature nanotechnology (2016)

[18] Yu, Haiming, G. Duerr, R. Huber, M. Bahr, T. Schwarze, F. Brandl, and D. Grundler. "Omnidirectional spin-wave nanograting coupler." Nature communications 4 (2013).

[19] Papp, A., W. Porod, and G. Csaba. "Hybrid yttrium iron555 garnet-ferromagnet structures for spin-wave devices." Journal of Applied Physics 117, no. 17 (2015): 17E101.

[20] Vogel, Marc, Andrii V. Chumak, Erik H. Waller, Thomas Langner, Vitaliy I. Vasyuchka, Burkard Hillebrands, and Georg von Freymann. "Optically reconfigurable magnetic materials." 560 Nature Physics 11, no. 6 (2015): 487-491.

[21] Albisetti, E., D. Petti, M. Pancaldi, M. Madami, S. Tacchi, J. Curtis, W. P. King et al. "Nanopatterning reconfigurable magnetic landscapes via thermally assisted scanning probe lithography." Nature nanotechnology (2016).

[22] Jungfleisch, Matthias B., Wei Zhang, Wanjun Jiang, Houchen Chang, Joseph Sklenar, Stephen M. Wu, John E. Pearson et al. "Spin waves in micro-structured yttrium iron garnet nanometerthick films." Journal of Applied Physics 117, no. 17 (2015): $17 \mathrm{D} 128$.

[23] Nikitin, Andrey A., Alexey B. Ustinov, Alexander A. Semenov, Andrii V. Chumak, Alexander A. Serga, Vitaliy I. Vasyuchka, Erkki Lahderanta, Boris A. Kalinikos, and Burkard Hillebrands. "A spin-wave logic gate based on a width-modulated dynamic magnonic crystal." Applied Physics Letters 106, no. 10 (2015):575 102405.

[24] Brandl, F., K. J. A. Franke, T. H. E. Lahtinen, S. van Dijken, and D. Grundler. "Spin waves in CoFeB on ferroelectric domains combining spin mechanics and magnonics." Solid State Communications 198 (2014): 13-17.

[25] Madami, M., D. Chiuchi, G. Carlotti, and L. Gammaitoni. "Fundamental energy limits in the physics of nanomagnetic binary switches." Nano Energy 15 (2015): 313-320.

[26] Papp, Adam, Gyorgy Csaba, George I. Bourianoff, and Wolfgang Porod. "Spin-wave-based computing devices." In 14th IEEE International Conference on Nanotechnology. 2014.

[27] Breitkreutz, Stephan, Adam Papp, Eugen Egel, Christian Meier, Cenk Yilmaz, Leonhard Heiss, Wolfgang Porod, and Gyorgy Csaba. "Design of on-chip readout circuitry for spin-wave devices." IEEE Magnetics Letters (2016).

[28] Demidov V E, Urazhdin S, Liu R, et al. Excitation of coherent propagating spin waves by pure spin currents. Nature communications, 2016, 7. .)

[29] Khitun, Alexander, and Kang L. Wang. "Nano scale computational architectures with Spin Wave Bus." Superlattices and Microstructures 38, no. 3 (2005): 184-200.

[30] Khitun, Alexander, Dmitri E. Nikonov, Mingqiang Bao, Kosmas Galatsis, and Kang L. Wang. "Efficiency of spin-wave bus for information transmission." IEEE Transactions on Electron Devices 54, no. 12 (2007): 3418.

[31] Yu, Haiming, O. d?Allivy Kelly, V. Cros, R. Bernard, P. Bortolotti, A. Anane, F. Brandl, F. Heimbach, and D. Grundler. "Approaching soft X-ray wavelengths in nanomagnet-based microwave technology." Nature communications 7 (2016).

[32] Klingler, Stefan, Philipp Pirro, Thomas Brcher, Britta Leven, Burkard Hillebrands, and Andrii V. Chumak. "Spin-wave logic devices based on isotropic forward volume magnetostatic waves." Applied Physics Letters 106, no. 21 (2015): 212406.

[33] Schneider, T., A. A. Serga, B. Leven, B. Hillebrands, R. L. Stamps, and M. P. Kostylev. "Realization of spin-wave logic gates." Applied Physics Letters 92, no. 2 (2008): 022505.

[34] Chumak, Andrii V., Alexander A. Serga, and Burkard Hillebrands. "Magnon transistor for all-magnon data processing." Nature communications 5 (2014).

[35] Sadovnikov, Alexandr V., Carl S. Davies, Sergey V. Grishin, V. V. Kruglyak, D. V. Romanenko, Yu P. Sharaevskii, and S. A. Nikitov. "Magnonic beam splitter: The building block of parallel magnonic circuitry." Applied Physics Letters 106, no. 19 (2015): 192406 .

[36] Nikonov, Dmitri E., and Ian A. Young. "Overview of beyondCMOS devices and a uniform methodology for their benchmarking." Proceedings of the IEEE 101, no. 12 (2013): 2498-2533.

[37] Csaba, G., A. Papp, and W. Porod. "Spin-wave based realization of optical computing primitives." Journal of Applied Physics 115, no. 17 (2014): $17 \mathrm{C} 741$

[38] Toedt, Jan-Niklas, Mark Mundkowski, Detlef Heitmann, Stefan Mendach, and Wolfgang Hansen. "Design and construction of a spin-wave lens." Scientific Reports 6 (2016).

[39] Jeon, Dongsuk, Mingoo Seok, Chaitali Chakrabarti, David Blaauw, and Dennis Sylvester. "A super-pipelined energy efficient subthreshold $240 \mathrm{MS} / \mathrm{s}$ FFT core in $65 \mathrm{~nm}$ CMOS." IEEE Journal of Solid-State Circuits 47, no. 1 (2012): 23-34.

[40] Kozhevnikov, Alexander, Frederick Gertz, G. Dudko, Y. Filimonov, and A. Khitun. "Pattern recognition with magnonic holographic memory device." Applied Physics Letters 106, no. 14 (2015): 142409

[41] Khivintsev, Y., Ranjbar, M., Gutierrez, D., Chiang, H., Kozhevnikov, A., Filimonov, Y. and Khitun, A., 2016. Prime Factorization Using Magnonic Holographic Device. arXiv preprint arXiv:1605.02127.

[42] Eshaghian-Wilner, Mary M., Alexander Khitun, and Kang L. Wang. "Spin-wave architectures." U.S. Patent 7,535,070, issued May 19, 2009.

[43] Papp, A., Porod. W., Csaba, G.: Nanoscale spectrum analyzer based on spin-wave interference, to be submitted to Scientific Reports (2016)

[44] Kaka, Shehzaad, Matthew R. Pufall, William H. Rippard, Thomas J. Silva, Stephen E. Russek, and Jordan A. Katine. "Mutual phase-locking of microwave spin torque nano-oscillators." 
Nature 437, no. 7057 (2005): 389-392.

[45] Nikonov DE, Csaba G, Porod W, Shibata T, Voils D, Hammerstrom D, Young IA, Bourianoff GI. Coupled-Oscillator Associative Memory Array Operation for Pattern Recognition. IEEE Journal on Exploratory Solid- State Computational Devices and Circuits. 2015 Dec;1:85-93.

[46] Csaba, Gyorgy, Adam Papp, Wolfgang Porod, and Ramazan Yeniceri. "Non-boolean computing based on linear waves and oscillators." In 2015 45th European Solid State Device Research Conference (ESSDERC), pp. 101-104. IEEE, 2015.

[47] Horvath, Andras, Gyorgy Csaba, and Wolfgang Porod. "Dy-660 namic coupling of spin torque oscillators for associative memories." In 2014 14th International Workshop on Cellular Nanoscale Networks and their Applications (CNNA), pp. 1-2. IEEE, 2014.

[48] Locatelli, Nicolas, Vincent Cros, and Julie Grollier. "Spintorque building blocks." Nature materials 13, no. 1 (2014): 11-20.665

[49] Chang, Houchen, Peng Li, Wei Zhang, Tao Liu, Axel Hoffmann, Longjiang Deng, and Mingzhong Wu. "Nanometer-thick yttrium iron garnet films with extremely low damping." IEEE Magnetics Letters 5 (2014): 1-4.

[50] Hauser, Christoph, Tim Richter, Nico Homonnay, Christian670 Eisenschmidt, Mohammad Qaid, Hakan Deniz, Dietrich Hesse, Maciej Sawicki, Stefan G. Ebbinghaus, and Georg Schmidt. "Yttrium Iron Garnet Thin Films with Very Low Damping Obtained by Recrystallization of Amorphous Material." Scientific reports 6 (2016).

[51] Che, P., Zhang, Y., Liu, C., Tu, S., Liao, Z., Yu, D., Vetro, F.A., Ansermet, J.P., Zhao, W., Bi, L. and Yu, H., 2016. Shortwavelength Spin Waves in Yttrium Iron Garnet Micro-Channels on Silicon. IEEE Magnetics Letters 2016 (early access)

[52] Schoen, Martin AW, Danny Thonig, Michael L. Schneider, T. J. Silva, Hans T. Nembach, Olle Eriksson, Olof Karis, and Justin M. Shaw. "Ultra-low magnetic damping of a metallic ferromagnet." Nature Physics (2016).

[53] Yu, Haiming, R. Huber, T. Schwarze, F. Brandl, T. Rapp, P. Berberich, G. Duerr, and D. Grundler. "High propagating velocity of spin waves and temperature dependent damping in a CoFeB thin film." Applied Physics Letters 100, no. 26 (2012): 262412 .

[54] Wu, Mingzhong. M-Type barium hexagonal ferrite films. INTECH Open Access Publisher, 2012.

620 [55] Madami, M., S. Bonetti, G. Consolo, S. Tacchi, G. Carlotti, G. Gubbiotti, F. B. Mancoff, Mazher Ahmed Yar, and Johan kerman. "Direct observation of a propagating spin wave induced by spin-transfer torque." Nature nanotechnology 6, no. 10 (2011): 635-638.

[56] Demidov, Vladislav E., Sergei Urazhdin, and Sergej O. Demokritov. "Direct observation and mapping of spin waves emitted by spin-torque nano-oscillators." Nature materials 9 , no. 12 (2010): 984-988.

[57] Urazhdin, S., V. E. Demidov, H. Ulrichs, T. Kendziorczyk, T. Kuhn, J. Leuthold, G. Wilde, and S. O. Demokritov. "Nanomagnonic devices based on the spin-transfer torque." Nature nanotechnology 9, no. 7 (2014): 509-513.

[58] Balinsky, Michael, Mohammad Haidar, Mojtaba Ranjbar, Philipp Drrenfeld, Afshin Houshang, Andrei Slavin, and Johan kerman. "Modulation of the Spectral Characteristics of a NanoContact Spin-Torque Oscillator via Spin Waves in an Adjacent Yttrium-Iron Garnet Film." IEEE Magnetics Letters 7 (2016): $1-4$.

[59] Cherepov S, Khalili Amiri P, Alzate JG, Wong K, Lewis M, Upadhyaya P, Nath J, Bao M, Bur A, Wu T, Carman GP. K. Wang Electric-field-induced spin wave generation using multiferroic magnetoelectric cells. Applied Physics Letters. 2014 Feb 24;104(8):082403.

[60] Demidov, V. E., M. P. Kostylev, Karsten Rott, Jana Mnchenberger, Gnter Reiss, and S. O. Demokritov. "Excitation of shortwavelength spin waves in magnonic waveguides." Applied Physics Letters 99, no. 8 (2011): 082507.

[61] Van de Wiele, Ben, Sampo J. Hmlinen, Pavel Bala, Federico Montoncello, and Sebastiaan van Dijken. "Tunable short- wavelength spin wave excitation from pinned magnetic domain walls." Scientific reports 6 (2016).

62] Li, Peng, Tao Liu, Houchen Chang, Alan Kalitsov, Wei Zhang, Gyorgy Csaba, Wei Li et al. "Spin-orbit torque-assisted switching in magnetic insulator thin films with perpendicular magnetic anisotropy." Nature Communications 7 (2016).

[63] Jungfleisch, M. B., V. Lauer, R. Neb, A. V. Chumak, and B. Hillebrands. "Improvement of the yttrium iron garnet/platinum interface for spin pumping-based applications." Applied Physics Letters 103, no. 2 (2013): 022411.

[64] Chumak, A. V., A. A. Serga, M. B. Jungfleisch, R. Neb, D. A. Bozhko, V. S. Tiberkevich, and B. Hillebrands. "Direct detection of magnon spin transport by the inverse spin Hall effect." Applied Physics Letters 100, no. 8 (2012): 082405.

[65] Zhang, Xufeng, Chang-Ling Zou, Liang Jiang, and Hong X. Tang. "Cavity magnomechanics." Science advances 2, no. 3 (2016): e1501286.

[66] Patil, Ranjit A., Chiung-Wu Su, Chin-Jung Chuang, ChienChih Lai, Yung Liou, and Yuan-Ron Ma. "Terahertz spinwave waveguides and optical magnonics in one-dimensional $\mathrm{NiO}$ nanorods." Nanoscale (2016). 